\title{
Sustained polymer membranes fabricated by nanoimprint lithography
}

\author{
Helmut Schift \\ Paul Scherrer Institut \\ Laboratory for Micro- and Nanotechnology \\ 5232 Villigen PSI, Switzerland \\ E-mail: helmut.schift@psi.ch \\ Sandro Bellini \\ University of Applied Sciences Nordwestschweiz \\ Institute of Nanotechnological Applications \\ in Polymers \\ 5210 Windisch, Switzerland
}

\author{
Uwe Pieles \\ University of Applied Sciences Nordwestschweiz \\ Department of Chemistry and Nanotechnology \\ 4132 Muttenz, Switzerland

\section{Jens Gobrecht} \\ Paul Scherrer Institut \\ Laboratory for Micro- and Nanotechnology \\ 5232 Villigen PSI, Switzerland \\ and \\ University of Applied Sciences Nordwestschweiz \\ Institute of Nanotechnological Applications \\ in Polymers \\ 5210 Windisch, Switzerland
}

\begin{abstract}
Perforated polymer membranes are fabricated using nanoimprint lithography and a sacrificial layer technique. The membranes with micrometer-sized pores are partially released from the substrate by locally dissolving the underlying layer. Thus, large arrays with interconnected pores can be fabricated. This process can be applied to a wide selection of polymeric materials, and has the potential to be extended to submicrostructures. (ㅇ 2006 Society of Photo-Optical Instrumentation Engineers. [DOI: 10.1117/1.2172993]
\end{abstract}

Subject terms: nanoimprint lithography; sacrificial layer; membrane; thermoplastic polymer; lift-off resist; support column.

Paper NP-10 received Mar. 1, 2005; revised manuscript received May 1, 2005; accepted for publication Aug. 1, 2005; published online Feb. 13, 2006.

\section{Introduction}

\subsection{Polymer Membranes Made by Sacrificial Layer Techniques}

Micro- and nanoporous membranes are used as filters for fluids, ${ }^{1}$ photonic crystals, ${ }^{2}$ stencil masks, ${ }^{3}$ templates for cell culturing and biological testing, ${ }^{4-7}$ etc. They often take advantage of the high mechanical stability of membranes fabricated from thin films of $\mathrm{Si}_{3} \mathrm{~N}_{4}, \mathrm{SiO}_{2}$ or other solids, and span over millimeters. ${ }^{8}$ In many cases, the fabrication by silicon micromachining is too costly, particularly if singleuse sieves are needed, e.g., for biological experiments. The large surfaces of some square millimeters are often not required, and a surface membrane structure can have advantages over a free-standing sieve, e.g., when integrated into a microfluidic device. ${ }^{9}$ Polymers are good candidates for such low-cost devices and have proven to be suitable for fluidics and optics. ${ }^{10}$ They are biocompatible and can be processed using molding techniques. ${ }^{11-13}$ However, their low mechanical stability and their low resistance to chemicals, as well as the low selectivity between different polymers when used in stacks, are often a challenge for processing.

In this work, we show how nanoimprint lithography (NIL) in combination with a simple bilayer process based

$1537-1646 / 2006 / \$ 22.00$ ๑ 2006 SPIE on a polymeric sacrifical layer can be used to generate freestanding polymer membranes (see Fig. 1). NIL is a technique where a thin layer of a resist is structured by molding and not, as is commonly used, by optical exposure. ${ }^{14}$ With

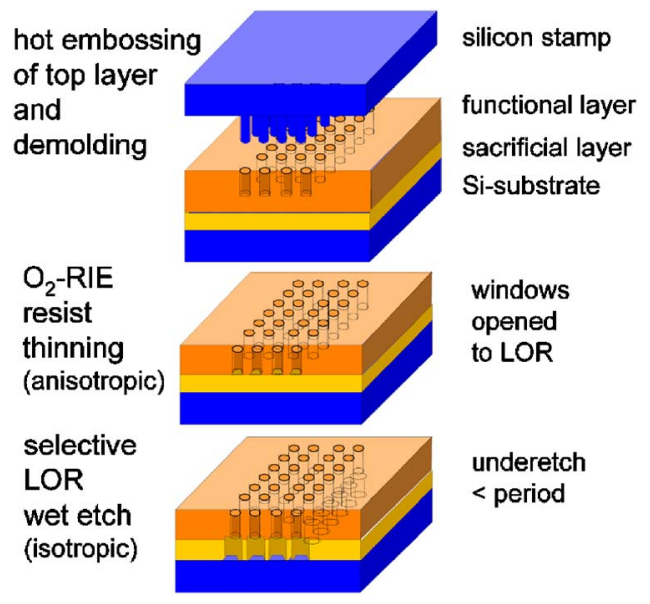

Fig. 1 Schematic view of a bilayer process with a structured functional layer on top of a sacrificial layer (LOR). The pores are generated via nanoimprint, followed by pattern transfer using dry etching. By selective wet underetching of the sacrificial layer, undercuts are created below the functional layer. 


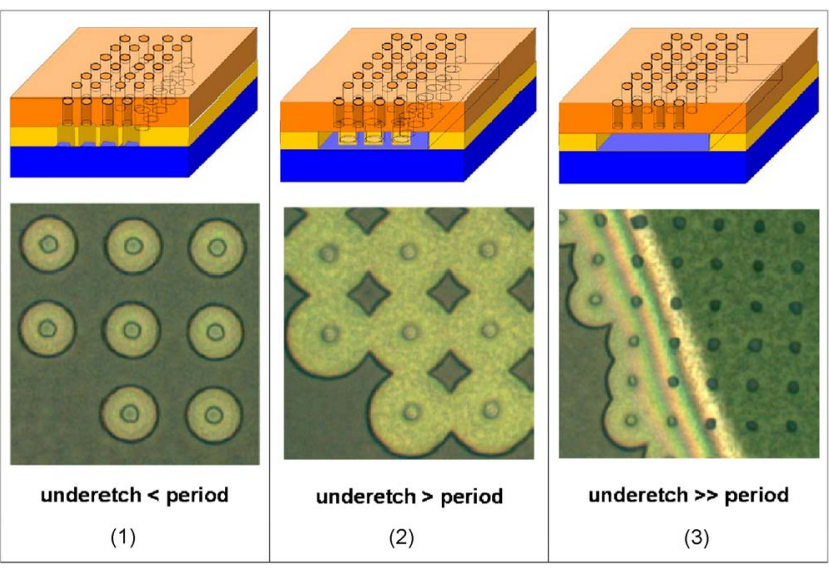

Fig. 2 Schematic view (top) and optical micrographs (bottom) for the three cases of (1) nonconnected, circular undercuts, (2) connected cavities with supporting columns, and (3) totally released, sagged membrane (PS $1 \mu \mathrm{m} / \mathrm{LOR} 1 \mu \mathrm{m}$, view size $30 \times 30 \mu \mathrm{m}^{2}$ ). The area between the pores and the sidewalls of the undercuts (bright) defines the membrane, and contrasts well the border and columns (dark) in diamond shape.

this technology, resolutions of below $10 \mathrm{~nm}$ were demonstrated. ${ }^{15}$ Because of its simple and large area processing capability, NIL is considered as one of the future low-cost fabrication methods for micro- and nanoapplications. ${ }^{16,17}$ As the sacrificial layer below the functional layer structured by NIL, a so-called lift-off resist (LOR) is used. ${ }^{18,19}$ Regular hole patterns were generated via NIL in the top layer and etched down to the sacrificial layer using $\mathrm{O}_{2}$-plasma. The underlying layer was dissolved (here referred to as "underetched") through the pores in a selective solvent (developer), thus creating an air gap. Several cases have to be distinguished for a regular array of holes with constant pitch and hole diameter (see Fig. 2)

1. If the LOR is slightly underetched, an undercut in the sidewalls is generated under the embossed holes. This serves as a means to discontinue an evaporated metal film for easy lift-off.

2. If the undercuts of two adjacent holes grow to more than half the length of the pore distance, the underlying cavities are connected. The resulting structure is a semidetached membrane which is sustained by a regular array of support columns.

3. If the LOR is underetched further, the columns shrink and finally disappear. The membrane is fully released from the substrate and is only suspended between the borders of the total structure. In this case, care has to be taken in the design of the device, so that the membrane does not sag between the borders and attaches to the substrate.

In this work, we focus on the fabrication of membranes described in case 2.

\subsection{Design Considerations for Free-Standing Polymer Membranes Supported by Columns}

A regular array of holes is defined by its period $p$ in $x$ and $y$ coordinates and by the hole diameter $a$ (see Fig. 3), which (a)

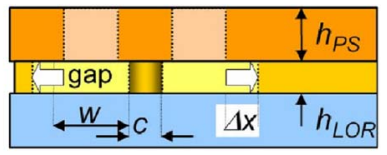

(b)

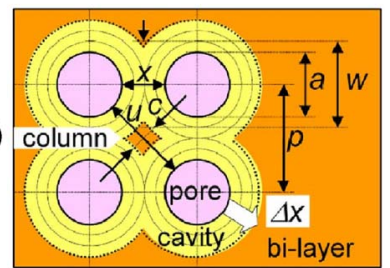

Fig. 3 Schematic view of the isotropic underetching of a regular array of holes: (a) side view, and (b) top view. The array is defined by its period $p$ in $x$ and $y$ coordinates and by the hole diameter $a$. During an underetch time $t$, the undercut grows by $\Delta x$, and the pores are interconnected by openings with width $w$ and a central column of size $c$.

in the following is described by the term $(p: a)$. The distance $x$ between two adjacent holes in the orthogonal matrix is given by $x=p-a$ and the distance $u$ between two holes in the diagonal direction by $u=p \cdot \sqrt{2}-a$. Therefore, case 2 will be achieved if the underetching distance $\Delta x$ is larger than $x / 2$, but still smaller than $u / 2$. The process time gap can be calculated for this case, assuming that for a LOR thickness smaller than the pore diameter, the underetching time $t$ is in first approximation proportional to the underetching distance. Stable columns will only be generated if the time $t$ is within a process window

$t_{\min }=x /\left(2 \cdot r_{\mathrm{LOR}}\right) \leqslant t=\Delta x /\left(2 \cdot r_{\mathrm{LOR}}\right) \leqslant t_{\max }=u /\left(2 \cdot r_{\mathrm{LOR}}\right)$.

From

$t_{\max }=u /\left(2 \cdot r_{\mathrm{LOR}}\right)=x /\left(2 \cdot r_{\mathrm{LOR}}\right) \cdot F_{\mathrm{diag}}=t_{\mathrm{min}} \cdot F_{\mathrm{diag}}$,

a factor $F_{\text {tol }}=\left(F_{\text {diag }}-1\right)$ can be calculated, which defines the process window from the first interconnection of orthogonal pores to the moment, when diagonal pores interconnect, i.e., at the full dissolution of the columns. For a large $(p: a)$ ratio of (10:2), $F_{\text {tol }}$ would be about $50 \%$ of the minimum etching time, and for the case of (5:3) around $100 \%$. This means that for small values of $F_{\text {tol }}$, the etching time has to be controlled within tight tolerances, and therefore longer underetching times are useful. In the real case, this factor is further reduced to assure a minimum size of the columns and to account for the tolerances of underetching parameters. By assuming for a column size $c$ a minimum aspect ratio of 1 , for $p=10 \mu \mathrm{m}, a=2 \mu \mathrm{m}$, and $c=h_{\mathrm{LOR}}=1 \mu \mathrm{m}$, this would result in $F_{\text {tol }} \approx 37 \%$. For $p=5 \mu \mathrm{m}, a=3 \mu \mathrm{m}$, and $c=h_{\mathrm{LOR}}=0.5,1,2 \mu \mathrm{m}, F_{\text {tol }}$ accounts to $F_{\mathrm{tol}}=75,50$, and $0 \%$, respectively. Therefore, for $h_{\mathrm{LOR}}=2 \mu \mathrm{m}$, the minimum aspect ratio 1 would already be generated at $t_{\min }$, when the pores start to interconnect. For $h_{\mathrm{LOR}}=1 \mu \mathrm{m}$, at $1.5 \cdot t_{\min }$, the window $w$ between adjacent pores would be $3 \mu \mathrm{m}$ wide. These calculations show the possibilities but also the fabrication limits, of membranes with interconnecting pores. 


\section{Experimental Design}

The bilayer process is based on lift-off resist (LOR) from Microchem ${ }^{\mathrm{TM}}$, routinely used for generating undercuts in resist profiles used for lift-off of metals. This resist, cyclopentanon dissolved in 1-methoxy-2-propanol, can be selectively dissolved in tetramethyl-ammonium-hydroxide, against common polymers and photoresists, and up to now has shown resolutions down to $100 \mathrm{~nm} .{ }^{18}$ According to the product description, ${ }^{20}$ the LOR layer develops nearly isotropically to produce bilayer re-entrant resist profiles. The amount of undercut can be controlled by the LOR prebake conditions, as well as through the choice of developer, develop time, and develop mode. Because for the LOR B type the $T_{g}$ varies from 160 to $200{ }^{\circ} \mathrm{C}$, depending on prebake conditions, a long prebake of $3 \mathrm{~min}$ at $190{ }^{\circ} \mathrm{C}$ on a hot plate was chosen to achieve a high $T_{g}$ and a low etching rate almost independent from further heat treatment. Two advantages were expected. First, the $T_{g}$ would be sufficiently high so that no deformation would occur in the LOR during top layer embossing. Second, the develop time would not be dependent from parameter variations during the NIL heating process. Different cases have been considered by the LOR manufacturer by providing LORs with high sensitivity to the developer and by adding wetting agents (detergents). In one of the foreseen applications, a sacrificial layer thickness $h_{\mathrm{LOR}}$ is needed between 1 and several $\mu \mathrm{ms}$. LOR $10 \mathrm{~B}$ and $30 \mathrm{~B}$ were spincoated on untreated silicon wafer substrates. Multiple coatings of LOR $10 \mathrm{~B}$ in 1- $\mu \mathrm{m}$ steps were possible after the prebake of each coating step. As developer for the microstructured pores, the Microposit MF319 (from Microchem ${ }^{\mathrm{TM}}$ ) was used.

As functional layer, polystyrene (PS from Polyscience $\mathrm{GmbH}$ with a molecular weight of $125 \mathrm{~kg} / \mathrm{kmol}$, dissolved in toluene) was chosen because of its excellent optical and physiological properties. In contrast to polymethyl methacrylate (PMMA), its etching rate in oxygen plasma $\left(20 \mathrm{sccm} \mathrm{O}_{2}\right.$ with a power of $20 \mathrm{~W}$ at $20 \mathrm{mTorr}, 300 \mathrm{~K}$ in an Oxford Plasmalab 100 RIE machine) is significantly lower relative to LOR, which means that once the windows are opened, the etching continues at a higher speed in the LOR (PS $30 \mathrm{~nm} / \mathrm{min}$, PMMA $65 \mathrm{~nm} / \mathrm{min}$, LOR 10B $48 \mathrm{~nm} / \mathrm{min}){ }^{21}$ The $1-\mu \mathrm{m}$-thick thermoplastic film was spincoated on the LOR layer and baked at $170{ }^{\circ} \mathrm{C}$ for 1 min. By embossing a structured silicon stamp into the resist layer at $180^{\circ} \mathrm{C}$ at a pressure of 20 bar for $30 \mathrm{~min}$, the pillar pattern of the stamp was transferred into the PS. Then the structure was cooled down and demolded at $70{ }^{\circ} \mathrm{C}$. The thickness of the resist $h_{\mathrm{PS}}(1000 \mathrm{~nm})$ was chosen to have a sufficient lateral flow of material with the 800-nm-high structures.

Large orthogonal hole patterns (about $1 \mathrm{~mm}^{2}$ area) with pore diameters from 1.5 to $6 \mu \mathrm{m}$ and periods of 5 to $15 \mu \mathrm{m}$ were generated via nanoimprint $(800 \mathrm{~nm}$ deep). The $(p: a)$ combinations were $(5: 1.5),(5: 3),(10: 2)$, (10:4), (15:4), and (15:6) $\mu \mathrm{m}$. To open the windows to the LOR, the top layer was homogeneously etched down to the LOR using $\mathrm{O}_{2}$ plasma. The underlying LOR was dissolved in MF319 developer, which penetrated the pores and dissolved the LOR isotropically. Once the dissolution reaches the substrate at the bottom, the undercuts grow laterally into large cavities, in the case of round pores in circular

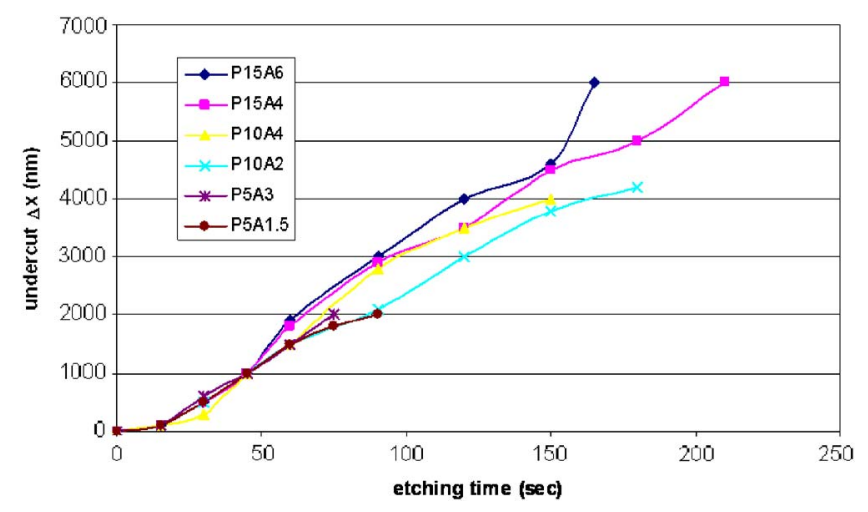

Fig. 4 Dependence of undercut distance on dissolution time for a $1-\mu \mathrm{m}$ sacrificial layer LOR 10B, for different combinations of periods $p$ and pore diameters $a$. Although very linear and well in agreement, as expected, for longer process times, structures with larger pore diameters are underetched faster, while long distances slow down underetching times.

shapes. For most experiments, dilutions of MF319 with water were prepared for slowing down the development process. Stopping the process was possible by extensive rinsing in deionized water. After drying in nitrogen, the water is completely removed from the cavities.

The progress of the underetching of the semidetached membrane was viewed under an optical microscope after each development step (see Fig. 2). Because the top layer is transparent, the underetch front wall can be clearly seen. Bright areas defining the membrane and the pore can be clearly distinguished from dark underetch borders and support columns in the LOR. However, due to the meniscus of the etch sidewall, the determination of the exact location is limited to about $0.5 \mu \mathrm{m}$, which was sufficient for the micrometer-sized structures in this research. Because of the $\mu \mathrm{m}$ thin gap between the top layer and substrate, the sagging of the membrane could also be detected optically.

\section{Results}

Most of the results were obtained with the regular array of micropores with the specifications mentioned before. Already at $h_{\mathrm{LOR}}=1 \mu \mathrm{m}$, all membranes with periods up to $15 \mu \mathrm{m}$ were free-standing without touching the substrate, as long as the membrane was sustained by the regular pattern of columns (see Fig. 2 bottom).

At a developer dilution of MF319/water of 3:2 (60\%), the underetching rates $r_{\mathrm{LOR}}$ range from $2.5 \mathrm{~nm} / \mathrm{s}$ for smaller to $5 \mathrm{~nm} / \mathrm{s}$ for larger periods. For the $(10: 2) \mu \mathrm{m}$ combination, a time $t_{\min }$ of about $13 \mathrm{~min}$ for half the distance etch was observed. In this case, the process window of $37 \%$ of $t_{\min }$ accounts to about $5 \mathrm{~min}$. To reduce the process time, the dilution was changed to $5: 1(85 \%)$. In this case, the underetching rates $r_{\text {LOR }}$ were enhanced and range from $22 \mathrm{~nm} / \mathrm{s}$ to $36 \mathrm{~nm} / \mathrm{s}$ (see Fig. 4). The time $t_{\min }$ for the $(10: 2) \mu \mathrm{m}$ half distance etch was about $2.5 \mathrm{~min}$, which results in a process window of about $1 \mathrm{~min}$. Although for combinations of smaller periods and pore diameters the underetching rate slows down, no limitation for the application of this technique for smaller diameters of below $1 \mu \mathrm{m}$ could be seen. 


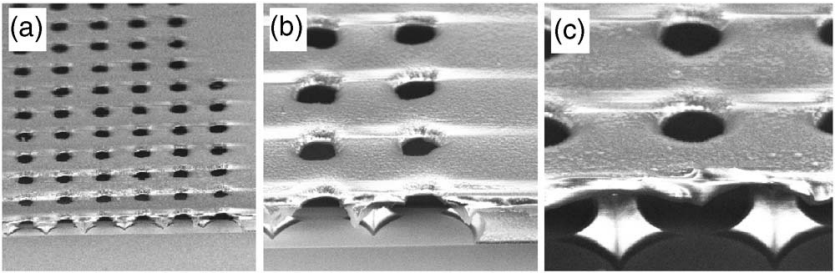

Fig. 5 SEM micrographs of a pore array in 1- $\mu \mathrm{m}$-thick polystyrene supported by $2-\mu \mathrm{m}$-high pillars with $3-\mu \mathrm{m}$ hole diameter and $5-\mu \mathrm{m}$ period: (a) overview (cleaved sample), (b) detail with meniscus, and (c) columns.

The dissolution parameters were optimized to enable the connection between adjacent holes while maximizing the mechanical properties of the suspended membrane. From the SEM micrographs in Fig. 5, it can be seen that the columns do not have, as shown for smaller LOR thickness, vertical sidewalls. ${ }^{18}$ A concave meniscus occurs during the etching of the sacrificial layer. This is a result of the inhomogeneous underetching in the vertical direction by the developer due to a reduced attack at the top and bottom layer. At the borders of the membranes, the meniscus can be clearly seen, with a lateral variation of about half the thickness of the LOR. In the center of the pore array, this effect leads to columns with large supporting areas but a thin center. They have the shape of a truncated pyramid mirrored at the top with concavely rounded flanks. Even when the underlying cavities are connected and the developer can further penetrate, the corners remain sharp. This clearly demonstrates the selectivity of the process.

Structures with $h_{\text {LOR }}$ of several $\mu \mathrm{ms}$ were fabricated. The underetching profile of multiple coatings of LOR 10B did not exhibit apparent asymmetry, and profiles looked similar to a single coating of LOR 30B with the same 2 or $3 \mu \mathrm{m}$ thickness. However, SEM micrographs reveal that for cases where the PS membrane was intentionally detached from the surface, column trunks remained on the surface (see Fig. 6). The columns are sometimes exactly broken in the center, at the interface of the two superimposed LOR layers, suggesting that LOR 10B is not adhering well to the first layer of the cured LOR. Other trunks are elongated, resulting from a cold deformation due to the ripping forces.
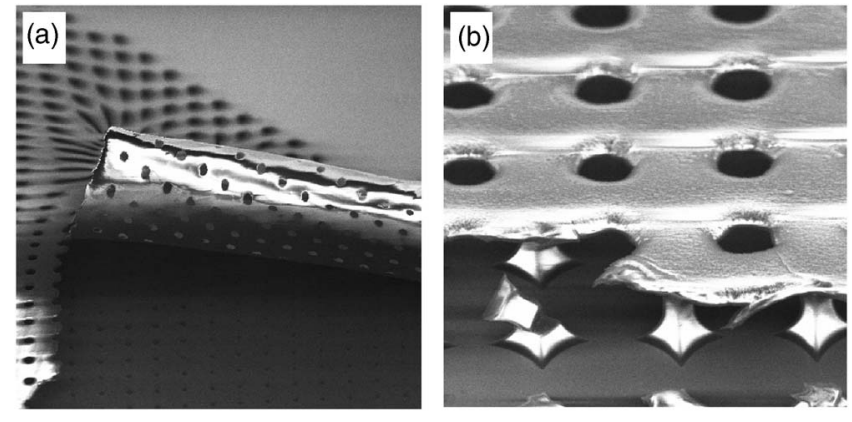

Fig. 6 SEM micrographs with effects seen for cases where the PS membrane was intentionally detached from the surface (distorsion due charging effects): (a) rolled membrane (SEM), and (b) membrane with broken column ( $2 \mu \mathrm{m}$ high).
In Fig. 2 it can be seen that at the border of a large array for 1- $\mu \mathrm{m}$-high gaps, the sagging of the membrane occurs already for distances less than $5 \mu \mathrm{m}$. The micrograph of a rolled membrane demonstrates the stability of polymer membranes (see Fig. 6). The membrane is clearly stable over several tens of $\mu \mathrm{ms}$, and suggests that polymer membranes can be fabricated with similar sizes as seen for $\mathrm{Si}_{3} \mathrm{~N}_{4}$ or $\mathrm{SiO}_{2}$.

\section{Discussion}

In our experiments, cases 1., 2., and 3. described in the introduction clearly could be reproduced. For the microstructured membranes, sustained perforated polymer membranes were developed, which consist of a regular sieve with an array of supporting columns. These columns serve as mechanical supports for the thin perforated polymer layer. Process parameters can be found for each combination of pore sizes and periods in this research. For connected membrane pores, the columns must remain large enough to support the membrane, and also if forces are present in the application, e.g., in fluidic transport. Furthermore, the profile of the underetched sidewall in the vertical direction has to be taken into account. A higher LOR thickness results in a larger maximal spanning range, but also in a larger meniscus undercut, thus limiting the minimum distance of the pores to about the height of the gap. No limitation for applications of this technique for smaller diameters of below $1 \mu \mathrm{m}$ could be seen. However, it is also obvious that the osmotic exchange through holes (principle of dialysis) of the fresh developer outside the pores with the developer saturated with dissolved LOR below the pores will be highly dependent on the pore diameter and LOR thickness.

The micrometer-sized structures shown in this research were achieved without the use of wetting agent in the developer, at reasonable process times of a few minutes, with tolerances acceptable for many applications. For cases with bad wetting conditions, e.g., small pores and low LOR thickness, the developer CD26 from Microchem ${ }^{\mathrm{TM}}$ is available. The development process could be stopped by rinsing in water, and the membranes were released by drying in nitrogen without the use of dry freezing or critical-point drying processes to reduce the effect of capillary forces. However, this has still to be proven for smaller gaps and for a larger membrane size-to-thickness ratio.

\section{Conclusion}

The fabrication process for supported polymer membranes has the advantage that it is versatile and fast, and by underetching through the holes of the patterned top layer, the area of detachment can be defined without any additional lithographic steps. It is a compliment to pattern reversal techniques, ${ }^{22,23}$ where the structure is first molded and then transferred to a substrate. Furthermore, the sagging of membranes is avoided and the size of the structured field is not limited. The top layer can be chosen from a range of thermoplastic materials with biological and optical properties optimized to the application, not limited to photosensitive materials, as would be necessary in the case of photolithographic patterning. In contrast to micromachined membranes, the sacrificial layer process circumvents the etching through the backside of the underlying substrate. 
However, the specific design necessary for the release and the double layer with two polymers with different chemical properties may restrict its use for some applications, e.g., for fluidic transport, the stability and adhesion of the membranes, and the compatibility of LOR with aqueous solutions is not yet proven. Here, other materials can be used as a sacrificial layer, ${ }^{24,25}$ and specific designs can be developed, which make it possible to transfer liquids to the membrane area. For example, by omitting single lines of pores, linear cavities can be generated with wide supporting walls. The membrane is then spanned over several pores and is supported by the walls for which the distance is higher than the dissolution distance. Although in this contribution we are focusing on the fabrication of micropores, it is expected that similar results can be achieved for hole diameters and periods much below $1 \mu \mathrm{m}$. Prospected applications are in optics and in biodevices, as free-standing polymer slab waveguides, ${ }^{25,26}$ as support for lipid membranes, ${ }^{7}$ or as filters or sieves in microfluidic applications. $^{27}$

Apart from being useful for membrane applications, the structures can also be used in their original sense, i.e., as intermediate structuring layers for lift-off. ${ }^{15,28}$ The undercuts can be generated in a way that cavities are interlinked, but the lift-off structures are still defined by the openings in the top layer.

\section{Acknowledgments}

The authors wish to thank E. Deckardt, F. Glaus, B. Haas, J. Lehmann, H. Sehr, A. Weber, and K. Vogelsang (PSI Villigen); S. Park (now at Louisiana State University); H. C. Scheer, and M. Wissen (University of Wuppertal); and H. Schulz (now at Zeiss, Oberkochem), for their help and valuable contributions. The research presented here was partially funded by the Swiss Federal Office for Science and Education in the framework of the EC-funded project $\mathrm{NaPa}$ (contract number NMP4-CT 2003-500120, OFES number 03.0424-4). The content of this work is the sole responsibility of the authors.

\section{References}

1. C. J. M. van Rijn, G. J. Veldhuis, and S. Kuiper, "Nanosieves with microsystem technology for microfiltration applications," Nanotechnology 9, 343-345 (1998).

2. S. J. McNab, N. Moll, and Y. A. Vlasov, "Ultra-low loss photonic integrated circuit with membrane-type photonic crystal waveguides," Opt. Express 11(22), 2927-2939 (2003).

3. J. Brugger, J. M. Berenschot, S. Kuiper, W. Nijdam, B. Otter, and M. Elwenspoek, "Resistless patterning of sub-micron structures by evaporation through nanostencils," Microelectron. Eng. 53, 403-405 (2000).

4. "Millicell cell culture plate inserts," Millipore Corp. Bedford, MA, see http://www.millipore.com.

5. J. M. Köhler, G. Mayer, S. Poser, T. Schulz, and A. Schober, "Chip elements for combinatorial chemistry, fluid processing and PCR," Proc. Micro System Technol. H. Reichl and A. Heuberger, Eds., 693698, VDE-Verlag, Berlin (1996).

6. E. E. Leary Swan, K. C. Popat, C. A. Grimes, and T. A. Desai, "Fabrication and evaluation of nanoporous alumina membranes for osteoblast culture," J. Biomed. Mater. Res. 72A, 288-295 (2005).

7. L. J. Heyderman, B. Ketterer, D. Bächle, F. Glaus, B. Haas, H. Schift, K. Vogelsang, J. Gobrecht, L. Tiefenauer, O. Dubochet, P. Surbled, and T. Hessler, "High volume fabrication of customised nanopore membrane chips," Microelectron. Eng. 67,68, 208-213 (2003).

8. A. Hierlemann, O. Brand, C. Hagleitner, and H. Baltes, "Microfabrication techniques for chemical/biosensors," Proc. IEEE 91(6), 839 (2003).

9. S. Metz, C. Trautmann, A. Bertsch, and P. Renaud, "Polyimide microfluidic devices with integrated nanoporous filtration areas manu- factured by micromachining and ion track technology," J. Micromech. Microeng. 14, 324-331 (2004).

10. D. Nilsson, S. Balslev, and A. Kristensen, "A microfluidic dye laser fabricated by nanoimprint lithography in a highly transparent and chemically resistant cyclo-olefin copolymer (COC)," J. Micromech. Microeng. 15, 296-300 (2005).

11. M. T. Gale, "Replication," in Micro-Optics, H. P. Herzig, Ed., Taylor \& Francis, London (1997).

12. M. Heckele and W. K. Schomburg, "Review on micro molding of thermoplastic polymers," J. Micromech. Microeng. 14, R1-R14 (2004).

13. H. Schift, J. Gobrecht, B. Satilmis, J. Söchtig, F. Meier, and W. Raupach, "Nanoreplication in a Network," Kunststoffe Plast Europe 94, 1-4 (2004).

14. R. W. Jaszewski, H. Schift, J. Gobrecht, and P. Smith, "Hot embossing in polymers as a direct way to pattern resist," Microelectron. Eng. 41,42, 575-578 (1998).

15. S. Y. Chou, P. R. Krauss, W. Zhang, L. Guo, and L. Zhuang, "Sub$10 \mathrm{~nm}$ imprint lithography and applications," J. Vac. Sci. Technol. B 15(6), 2897-2904 (1997).

16. "Alternative lithography-Unleashing the potential of nanotechnology," in Nanostructure Science and Technology, C. Sotomayor Torres, Ed., pp. 46-76, Kluwer Academic/Plenum Publishers, ISBN 0-306-47858-7 (2003).

17. L. J. Guo, "Recent progress in nanoimprint technology and its applications," J. Phys. D 37, R123-R141 (2004).

18. Y. Chen, K. Peng, and Z. Cui, "A lift-off process for high resolution patterns using PMMA/LOR resist stack," Microelectron. Eng. 73,74, 278-281 (2004).

19. P. Carlberg, M. Graczyk, E. L. Sawe, I. Maximov, M. Beck, and L. Montelius, "Lift-off process for nanoimprint lithography," Microelectron. Eng. 67,68, 203-207 (2003).

20. "LOR data sheets," Microchem ${ }^{\mathrm{TM}}$, see http://www.microchem.com/ products/lor.htm (Feb. 2005).

21. H. Schulz, M. Wissen, and H. C. Scheer, "Local mass transport and its effect on global pattern replication during hot embossing," Microelectron. Eng. 67,68, 657-663 (2003).

22. T. Borzenko, M. Tormen, G. Schmidt, and L. W. Molenkamp, "Polymer bonding process for nanolithography," Appl. Phys. Lett. 79(14), 2246-2248 (2001).

23. X. D. Huang, L. R. Bao, X. Cheng, L. J. Guo, S. W. Panga, and A. F. Yee, "Reversal imprinting by transferring polymer from mold to substrate," J. Vac. Sci. Technol. B 20(6), 2872-2876 (2002).

24. W. Li, J. O. Tegenfeldt, L. Chen, R. H. Austin, S. Y. Chou, P. A. Kohl, J. Krotine, and J. C. Sturm, "Sacrificial polymers for nanofluidic channels in biological applications," Nanotechnology 14, 1-6 (2003).

25. R. R. Panepucci, B. H. Kim, V. R. Almeida, and M. D. Jones, "Photonic crystals in polymers by direct electron-beam lithography presenting a phonic band gap," J. Vac. Sci. Technol. B 22(6), 33483351(2004).

26. H. Schift, S. Park, C. G. Choi, C. S. Kee, S. P. Han, K. B. Yoon, and J. Gobrecht, "Fabrication process for polymer photonic crystals using nanoimprint lithography," Nanotechnology 16, S261-S265 (2005).

27. L. J. Guo, X. Cheng, and C. F. Chou, "Fabrication of sizecontrollable nanofluidic channels by nanoimprinting and its application for DNA stretching," Nano Lett. 4, 69-73 (2004).

28. H. Schift, R. W. Jaszewski, C. David, and J. Gobrecht, "Nanostructuring of polymers and fabrication of interdigitated electrodes by hot embossing lithography," Microelectron. Eng. 46, 121-124 (1999).

Helmut Schift, Drlng, is a group leader at the laboratory of microand nanotechnology at the Paul Scherrer Institut (PSI) in Villigen, Switzerland. The focus of his work is the development of replication technologies.

Sandro Bellini, DiplChem, was engaged as a diploma student on this research project at PSI until January 2005, and is now a scientific assistant at the Institute of Nanotechnological Applications in Polymers (INKA).

Uwe Pieles is head of the nanotechnology laboratory of the Department of Chemistry at the University of Applied Sciences Nordwestschweiz, Switzerland.

Jens Gobrecht is head of the laboratory of micro- and nanotechnology at PSI, and since 2004 has been head of INKA, a joint institute of PSI and the University of Applied Sciences Nordwestschweiz. 